\title{
Cerebrovascular mediated subclinical brain injury - interaction between cardiovascular function, brain structure and cognitive function - study rationale, design and principal methods
}

\author{
Katarzyna Katulska', Andrzej Minczykowski², Jarosław Piskorski', Mateusz Wykrętowicz', \\ Marek Stajgis', Przemysław Guzik², Andrzej Wykrętowicz² \\ ${ }^{1}$ Department of Radiology, Poznan University of Medical Sciences, Poland \\ ${ }^{2}$ Department of Cardiology - Intensive Therapy, Poznan University of Medical Sciences, Poland \\ ${ }^{3}$ Institute of Physics, University of Zielona Góra, Poland
}

\begin{abstract}
Project entitled "Cerebrovascular mediated subclinical brain injury - interaction between cardiovascular function, brain structure and cognitive function- study rationale, design and principal methods" is a cross-sectional study in a group of older individuals with no apparent symptoms of stroke, transient ischemic attacks or cognitive impairment. The primary aim of the projects is further our understanding of the patomechanism of cerebrovascular disease associate brain injury as well as vascular cognitive impairment and give a better insight into the interplay between arterial wall, ventriculo-vascular coupling, wave reflection and pulsatility of the flow and how these interplays are mirrored by MRI neuroimaging. With our novel, interdisciplinary approach, we will define the methodology and build tools for those who want to follow in this challenging and non-standard direction.
\end{abstract}

Keywords: brain structure, cognitive function, arterial stiffness, arterio-vascular coupling, gray matter volume, white matter lesions.

\section{Introduction}

A population study performed with the magnetic resonance imaging (MRI) demonstrated high prevalence of salient changes in central nervous system [1, 2]. Among 3301 participants (mean age 65 years) who underwent MRI scanning and denied a history of stroke or transient ischemic attack only $4.4 \%$ had scans which were assigned a white matter grade of 0 , indicating that they were free of any abnormal signal in the white matter. Thus, most of this population showed degenerative changes, which may not be considered benign because they were associated with impaired cognitive and lower extremity function [2]. A large part of this problem is enhanced by vascular disease contributing to subclinical brain injury, silent brain infarction or clinically apparent stroke. The clinical spectrum of vascular contribution to cognitive impairment and dementia ranges from subclinical forms, being in fact risk factors for the development of clinically overt syndrome to overt stroke. Recently a broader terminology was introduced namely "vascular cognitive impairment" (VCI) in order to capture the entire spectrum of cognitive disorders linked to all forms of cerebral vascular brain injury [3]. Thus, $\mathrm{VCl}$ is regarded as syndrome with evidence of stroke or subclinical vascular brain injury based on clinical as well as neuroradiological imaging. Moreover, these changes are linked to impairment of $\geqslant 1$ cognitive domain.

Cerebrovascular disease brain injury (CVBI) ranges from overt stroke to microinfarction, brain athrophy or 
white matter lesions (WMLs). WMLs are highly correlated with white matter ischemic injury while gray matter volume changes are associated with both CVBI and Alzheimer [4]. In populations study WMLs as well as silent cerebral infarction are very common [5]. Nevertheless, several studies demonstrated the association of MRI markers of vascular brain injury with incident stroke, mild cognitive impairment, dementia, and mortality [6]. The risk factors for the development of CVBI include hypertension, atherosclerosis, tobacco, diabetes and atrial fibrillation [7].

Non-traditional risk factors for vascular brain injury Recently considerable interest was focused on nontraditional risk factors for the development of vascular cognitive impairment. Increased arterial stiffness or arteriosclerosis, represents a physiological part of aging that leads to diminished elastic properties of the media and increased arterial stiffness. This process may be further accelerated by hypertension, diabetes, renal insufficiency and other risk factors [8]. There are several methods, such as pulse wave velocity, digital volume pulse and pulse wave analysis or ultrasonographic indices of local arterial distensibility that allow the assessment of general, segmental or local arterial stiffness as well as central hemodynamics or pressure wave reflection. These measures as well as derived indices served as indicators of increased risk of cardiovascular complications independently of other well known risk factors $[9,10,11]$.

Henskens et al. [12] demonstrated that increased arterial stiffness measured by aortic pulse wave velocity (PWV) is associated with silent cerebral small-vessel disease in hypertensive patients. Kearney-Schwartz et al. [13] in a prospective study performed in 198 elderly (mean age 69 years) hypertensive patients showed that increased PWV, brachial endothelial function as well as increased intima-media thickness in carotid artery are associated with white matter hyperintensities and cognitive performance. There are several possible explanations for the pathophysiological link between increased arterial stiffness and microvascular brain injury. First, increased arterial stiffness leads to increased pulsatility in the ascending aorta which has a direct transfer to brain vasculature. Second, arterial stiffness is linked to endothelial dysfunction and oxidative stress both of which are associated with microvessel injury. Third, arterial stiffness leads to left ventricular hypertrophy, a risk factor for subclinical brain damage and dementia.

Cardiac diseases are associated with increased risk of cognitive impairment or dementia. It is obvious that chronic cerebral hypoperfusion due to severe carotid stenosis caused by atherosclerosis or significantly reduced cardiac output caused by left ventricular dysfunction may lead to cognitive impairment. Therefore, the majority of research concerning relationship between cardiac function and cerebral structure and cognitive performance was done in the population of subjects with chronic heart failure [14]. Recently, in preliminary studies we demonstrated that grey matter volume is correlated with some metrics of central hemodynamics as well as with arterial and left ventricular stiffness in a population of healthy subjects. Moreover, subclinical white matter lesions were present in those with higher arterial stiffness and increased values of aortic excess pressure $[15,16]$.

\section{Study hypothesis}

It is established that indices of subclinical atherosclerosis, wave reflection, arterial stiffness and cardiac dysfunction predict cardiovascular complications. Several investigators demonstrated that the characteristics of vascular beds obtained by these techniques are not synonymous and indicate different aspects of vascular status. Currently it is not known what exact contributions to cerebrovascular disease associated brain injury and/or vascular cognitive impairment may be attributed to each cardiovascular metric. We hypothesized that complementary assessment of vascular wall properties as well as cardiac structure and function combined with MRI evaluation of brain structure and cognitive testing allow to quantify the contribution of different cardio-arterial properties to subclinical brain injury and cognitive decline.

\section{Aim}

To perform complimentary assessment of arterial structure and function, left ventricular structure and function, peripheral and central hemodynamics in conjunction with brain magnetic resonance imaging. Additionally cognitive assessment will allow to performe analysis assessing interaction between cardiovascular and brain morphology metrics and cognitive performance.

The novelty input and influence of obtained results The novelty of our basic research is comprehensive evaluation of arterial and cardiac structure and function, together with central hemodynamics in the frame of neuroimaging and neuropsychologic evaluation of study subject. We hope that the obtained results will help to determine the optimal cardiovascular and brain 
structure metrics for testing in a future research performed in risk factors groups as well as subjects with established diagnosis of brain injury in order to prevent further deterioration or to monitor targeted therapy.

\section{Method and experimental plan}

Study group

The study group will include 230 volunteers, age $>60$ years with the perception of normal health, without apparent history of stroke, transient ischemic attack or cognitive decline. The exclusion criteria are: neoplasm, chronic kidney disease requiring hemodialysis, status post kidney or heart transplantation, atrial fibrillation, active infection, hyperthyreosis, implanted artificial device preventing MRI assessment (pacemakers, hip replacement etc.).

\section{Brain magnetic resonance imaging}

All brain examination will be performed on 1,5-Tesla MRI (Avanto Simens Medical System, Germany) using standard, circular, polarized volume head coil. MR images will be done in standard sequence. For the automatic separation of brain and nonbrain matter as well as the evaluation of white matter and gray matter brain volumes the FSL (University of Oxford) package as well as the SPM (Wellcome Trust Centre for Neuroimaging, London) and VBM (Structural Brain Mapping Group, University of Jena) Matlab toolboxes will be used. Additionally, use will be made of the MRICRON software to further analyze the regions of interest in the transformed images.

MR angiography of brain arteries will be made with Time of Flight sequence (TOF) without contrast injection.

For detailed brain structure segmentation into volumes and surfaces with the use of state-of-the-art algorithms the FreeSurfer (Laboratory for Computational Neuroimaging, Athinoula A. Martinos Center for Biomedical Imaging) software package will be used. White matter hyperintensities will be assessed with the use of the LST (Structural Brain Mapping Group, University of Jena) Matlab toolbox.

\section{Measurements}

All patients will be evaluated in a temperature controlled laboratory. Anthropometric measurements (height, weight, Waist-to-Hip Ratio [WHR]) will all be made according to standard guidelines. Brachial blood pressure will be obtained by an oscillometric method (M-6, Omron Healthcare Co, Ltd, Kyoto, Japan) in the supine position, after 10-minutes rest. Plasma glucose, cholesterol and creatinine levels will be obtained from subjects after an overnight fast. Six minute walk test will be performed.

\section{Cognitive function testing}

Psychological assessment

To account for psychological outcomes related to cardiovascular and brain changes we will measure subjective health status (Nottingham Health Profile) and cognitive function (neuropsychological battery) with reliable and validated measures. These assessments will be assigned, coded, and interpreted by trained psychologists. This approach will provide a comprehensive examination of outcomes resulting from identified medical conditions and enhance its unique interdisciplinary application. Findings will be particularly relevant to the discipline of psychocardiology and neuropsychology.

\section{Neuropsychological battery}

A comprehensive battery of neuropsychological assessments, including standard clinical neuropsychological instruments with established reliability and validity will be completed by all participants. We will evaluate attention-executive function with the Trail making B time to completion test, Digits Forward and Backwards, and Controlled Oral Word Association Test. Memory will be assessed with California Verbal Learning Test-II immediate recall and delayed recall. A total cognitive composite score will also be calculated. We will account for the premorbid functioning with WAIS-R vocabulary subtest. Composite measures will be computed for each test by converting raw scores to standardized z-scores and averaging them across the tests in each composite [17-22].

\section{Amount of body fat}

A bio-impedance analyzer (Bodystat 1500, Bodystat Ltd, UK) will be use to measure the fat content as a proportion of total body mass. Bio-impedance analysis (BIA) will be performed with a single frequency $(50$ $\mathrm{kHz}$ ) device.

Digital volume pulse analysis Measurement of the digital volume pulse (DVP) waveform will be performed by a photoplethysmographic method (Pulse Trace 2000, MicroMedical, UK). The Stiffness Index of the DVP $\left(\mathrm{SI}_{\mathrm{DVP}}\right)$ will obtained from the subject's body height (h) divided by the time between the systolic and diastolic peaks of the DVP. The $\mathrm{SI}_{\mathrm{DVP}}$ is 
an estimate of pulse wave velocity in large arteries and is regarded as a measure of large artery stiffness.

Peripheral and central pressure waves assessed by pulse wave analysis (PWA) for measurement of wave reflection and central hemodynamics The SphygmocorMx validated system (AtCor Medical, software version 7.0) will be used for measurement of the wave reflection indices and central hemodynamics. Non-invasive beat-to-beat finger arterial blood pressure will be recorded continuously with the use of a volumeclamp photoplethysmograph (Portapres 2, FMS, The Netherlands) with the sensor on the middle finger of the right hand. Calculations of mean blood pressure (MBP), heart rate (HR), total peripheral resistance (TPR) will be made using the Modelflow algorithm.

\section{Carotid Intima-Media Thickness and local carotid stiffness evaluation}

All patients will undergo common carotid artery (CCA) measurements with a novel system (ArtLab, Esaote, Italy) based on high-resolution echotracking technology (WallTrack system), including the use of a $128 \mathrm{RF}$ line multiarray. This novel technology gives access to all major mechanical parameters. The system employs dedicated software (RF-data technology involving RF Quality Intima-media Thickness (RFQIMT) and RF Quality Arterial Stiffness (RFQAS); Esaote Medical Systems).

\section{Echocardiography}

Patients will be imaged using a commercially available system (Vivid 9, General Electric-Vingmed). Data acquisition will be performed using a 3.5 $\mathrm{MHz}$ transducer, at a depth of $16 \mathrm{~cm}$ in the parasternal and apical views. Standard M-mode and 2D images will be obtained and analysis will be performed off-line. Peak systolic longitudinal strain and strain rate will be assessed on apical two-chamber, four-chamber, and long-axis views using speckle tracking analysis. Left ventricular end systolic stiffness, effective arterial elastance and ventriculararterial coupling will be estimated with the single beat technique. Total arterial compliance (TAC) is an additional measure of arterial pulsatile load on the heart and it will be estimated by dividing SV by the pulse pressure (PP).

\section{Conclusion and dissemination of the results}

It is expected that the work proposed will further our understanding of the patomechanism of cerebrovascu- lar disease associated brain injury as well as vascular cognitive impairment and give a better insight into the interplay between arterial wall, ventriculo-vascular coupling, wave reflection and pulsatility of the flow and how these interplays are mirrored by MRI neuroimaging. Also, with our novel, interdisciplinary approach, we will define the methodology and build tools for those who want to follow in this challenging and nonstandard direction.

\section{References}

1. Longstreth WT Jr, Bernick C, Manolio TA, Bryan N, Jungreis CA, Price TR. Lacunar infarcts defined by magnetic resonance imaging of 3660 elderly people: the Cardiovascular Health Study. Arch Neurol. 1998;55:1217-25.

2. Longstreth WT Jr, Manolio TA, Arnold A, Burke GL, Bryan $\mathrm{N}$, Jungreis $\mathrm{CA}$, Enright PL, O'Leary D, Fried L. Clinical correlates of white matter findings on cranial magnetic resonance imaging of 3301 elderly people:the Cardiovascular Health Study. Stroke. 1996;27:1274-82.

3. Hachinski V, ladecola C, Petersen RC, Breteler MM, Nyenhuis DL, Black SE, Powers WJ, DeCarli C, Merino JG, Kalaria RN, Vinters HV, Holtzman DM, Rosenberg GA, Wallin A, Dichgans M, Marler JR, Leblanc GG. National Institute of Neurological Disorders and Stroke - Canadian Stroke Network vascular cognitive impairment harmonization standards. Stroke. 2006;37:2220-41.

4. Fernando MS, Simpson JE, Matthews F, Brayne C, Lewis $C E$, Barber R, Kalaria RN, Forster G, Esteves F, Wharton SB, Shaw PJ, O'Brien JT, Ince PG; MRC Cognitive Function and Ageing Neuropathology Study Group. White matter lesions in an unselected cohort of the elderly: molecular pathology suggests origin from chronic hypoperfusion injury. Stroke. 2006;37:1391-8.

5. Das RR, Seshadri S, Beiser AS, Kelly-Hayes M, Au R, Himali JJ, Kase CS, Benjamin EJ, Polak JF, O'Donnell CJ, Yoshita M, D'Agostino RB Sr, DeCarli C, Wolf PA. Prevalence and correlates of silent cerebral infarcts in the Framingham offspring study. Stroke. 2008;39:2929-35.

6. Debette S, Beiser A, DeCarli C, Au R, Himali JJ, Kelly-Hayes M, Romero JR, Kase CS, Wolf PA, Seshadri S. Association of MRI markers of vascular brain injury with incident stroke, mild cognitive impairment, dementia, and mortality: the Framingham Offspring Study. Stroke. 2010; 41:600-6.

7. Das RR, Seshadri S, Beiser AS, Kelly-Hayes M, Au R, Himali JJ, Kase CS, Benjamin EJ, Polak JF, O'Donnell CJ, Yoshita M, D'Agostino RB Sr, DeCarli C, Wolf PA. Prevalence and correlates of silent cerebral infarcts in the Framingham offspring study. Stroke. 2008;39:2929-35.

8. O'RourkeMF, Hashimoto J, Mechanical factors in arterial aging. J Am Coll Cardiol. 2007;50:1-13.

9. Sutton-Tyrrell K, Najjar SS, Boudreau RM, Venkitachalam L, Kupelian V, Simonsick EM, Havlik R, Lakatta EG, Spurgeon $\mathrm{H}$, Kritchevsky S, Pahor M, Bauer D, Newman A for the Health $A B C$ Study et al. Elevated aortic pulse wave velocity, a marker of arterial stiffness, predicts cardiovascular events in well-functioning older adults. Circulation. 2005;111:3384-90. 
10. Boutouyrie P, Tropeano Al, Asmar R, Gautier I, Benetos A, Lacolley $P$, Laurent $S$. Aortic stiffness is an independent predictor of primary coronary events in hypertensive patients: a longitudinal study. Hypertension. 2002;39:10-15.

11. Meaume S, Benetos A, Henry OF, Rudnichi A, Safar ME. Aortic pulse wave velocity predicts cardiovascular mortality in subjects $>70$ years of age. Arterioscler Thromb Vasc Biol. 2001;21:2046-50.

12. Henskens LH, Kroon AA, van Oostenbrugge RJ, Gronenschild EH, Fuss-Lejeune MM, Hofman PA, Lodder J, de Leeuw PW. Increased aortic pulse wave velocity is associated with silent cerebral small-vessel disease in hypertensive patients. Hypertension. 2008;52:1120-6.

13. Kearney-Schwartz A, Rossignol P, Bracard S, Felblinger J, Fay R, Boivin JM, Lecompte T, Lacolley P, Benetos A, Zannad F. Vascular structure and function is correlated to cognitive performance and white matter hyperintensities in older hypertensive patients with subjective memory complaints. Stroke. 2009;40:1229-36.

14. Bhattacharya P, Bao F, Shah M, Ramesh G, Madhavan R, Khan $\mathrm{O}$. Left ventricular dysfunction is associated with grey matter injury: an in vivo brain MRI segmentation study. J Neurosci Sci. 2012;321:111-3.

15. Katulska K, Wykrętowicz M, Minczykowski A, Krauze T, Milewska A, Piskorski J, Marciniak R, Stajgis M, Wysocki H, Guzik P, Wykrętowicz A. Aortic excess pressure and arterial stiffness in subjects with subclinical white matter lesions. Int J Cardiol. 2014;1;172:269-70.

16. Katulska K, Wykrętowicz M, Minczykowski A, Krauze T, Milewska A, Piskorski J, Marciniak R, Stajgis M, Wysocki $H$, Guzik P, Wykrętowicz A. Grey matter volume in rela- tion to left ventricular end-systolic stiffness, arterial stiffness and central pressure augmentation. J Neurol Sci (submitted).

17. Delis DC, Kramer JH, Kaplan E, Ober BA. California Verbal Learning Test manual - adult version (research edition). The Psychological Corporation, New York 1987.

18. Hunt SM, McKenna SP, McEwen J, Williams J, Papp E. The Nottingham Health Profile: subjective health status and medical consultations. Soc Sci Med A. 1981;15:221-9.

19. Ruff RM, Light RH, Parker SB, Levin HS. Benton Controlled Oral Word Association Test: reliability and updated norms. Arch Clin Neuropsychol. 1996;11:329-38.

20. Reitan RM. Validity of the Trail Making Test as an indicator of organic brain damage. Percept Mot Skills. 1958; 8:271-6.

21. Wechsler D. Wechsler Adult Intelligence Scale. Manual. The Psychological Corporation, New York 1955.

22. Wrześniewski K. Development of a Polish version of the Nottingham Health Profile. Quality of Life Research. 2000;25:20-2.

Correspondence address: Katarzyna Katulska Department of Radiology Poznan University of Medical Sciences 49 Przybyszewskiego Street 60-355 Poznań, Poland phone: +48 501698777 email: katarzyna_katulska@op.pl 